\title{
Study Of Cardic Valvular Calcification In ESRD Patients On Regular Hemodialysis (A Single Center Study) \\ Abdel-Bassit El Shaarawy ${ }^{1}$, Mona Hosny ${ }^{1}$, Manar Raafat ${ }^{2}$ and Nelly Gendy ${ }^{2}$ \\ ${ }^{1}$ Internal Medicine Department, Faculty of Medicine, Ain Shams University; \\ ${ }^{2}$ Internal Medicine Department, Theodor Bilharze Institute.
}

\begin{abstract}
:
Cardiac valve calcification are common among patients with chronic kidney disease (CKD). Risk factors include alterations in calcium and phosphorus metabolism, elevated calcium phosphorus product and persistent elevations in plasma parathyroid hormone (PTH) Echocardiography is a simple and inexpensive method for detection of valvular calcifications as suggested by KDIGO guidelines.

60 Patients on regular HD constituted group A (36 males and 24 females) and 25 healthy volunteers constituted group B. Group A was subdivided into: Group I: 21 patients with no valvular calcification, group 2: 26 patients with aortic valve calcification and group 3: 13 patients with aortic and mitral valve calcification. For all, the following was done: clinical examination, serum $\mathrm{Ca}$, serum $\mathrm{P}$, serum albumin, serum creatinine, BUN and PTH level in blood. M-mode echo cardiography was done for all.
\end{abstract}

Age, duration of dialysis and duration of 1ry kidney disease was higher in group 2 and 3 compared to group $1(\mathrm{p}=0.0001)$. Calcium was higher in group 2 than group $1(\mathrm{p}=0.09)$ and group $3(\mathrm{p}=0.004)$ than group I phosphorus was higher in group 2 and 3 than group $1(\mathrm{P}=$ 0.001). $\mathrm{P}$ was higher in group 3 than group $2(\mathrm{p}=0.0001)$. Ca $\mathrm{P}$ was higher in group 2 and 3 than group $1(\mathrm{p}=0.0001)$, in group 3 than group $2(\mathrm{p}=0.01)$ PTH was higher in group 1 than group $2(\mathrm{p}=0.06)$. Cardiac dysfunction by echocardiography was least in group 1 , increasing in group 2 and being highest in group 3. It was found that calcified valve groups has taken higher doses of Calcium and Vitamin D3 .

We have to take care on prescribing $\mathrm{Ca}$ and vitamin $\mathrm{D} 3$ to ESRD patients on regular HD.

KEY WORDS: Valvular Calcification - Ca- P- PTH -Ca x P- HEMODIALYSIS. INTRODUCTION:

Patients with end-stage renal disease (ESRD) are frequently burdened with calcific valvular heart disease (Adragoa et al., 2004). Valvular involvement in ESRD is most commonly manifested as mitral annular calcification and aortic valve calcification. Both mitral and aortic valve calcification (MAC) occur more frequently and at younger age in those with ESRD than in those with normal renal function (Ernesto et al., 2003).

KDIGO provided a new definition of CKDMBD: a systemic disorder of mineral and bone metabolism due to $\mathrm{CKD}$, manifested by abnormalities of calcium, phosphorus, PTH or vitamin $\mathrm{D}$ metabolism, abnormalities in bone turnover, vascular or other soft-tissue calcification. The prevalence and severity of 
extraosseous calcification, increase as kidney function decreases (Uhlig et al., 2010).

KDIGO recommended that an echocardiogram can be used to detect the presence or absence of valvular calcification, as reasonable alternative to computed tomography based imaging in patients with CKD stages 3-5 (Moe et al., 2009).

\section{Aim of the Work}

To determine the frequency of valvular calcifications in hemodialysis patients using echocardiography and its relation to demographic and laboratory data.

\section{Patient and methods:}

The study was carried on 60 patients (group A) with ESRD on regular hemodialysis [24 females (40\%) and 36 males (60\%) mean age 49 years] and 25 healthy control subjects (group B), [11 females (44\%) and 14 males (56\%), mean age 49.32 years]. The study was conducted in hemodialysis unit of Theodor Bilharz research institute between July 2011 and January 2012. Group A patients started hemodialysis for more than six months prior to the study. Patients were dialyzed for 4 hours three times weekly using high flux filters and acetate solution as dialysate. All patients had arteriovenous fistula. We excluded from the study patients having rheumatic heart disease, congenital heart disease or rheumatic fever history.

Group A was divided into 3 subgroups according to the presence or absence of valvular calcification:

Group 1: Patients with no valvular calcification.
Group 2: Patients with aortic valve calcification only.

Group 3: Patients with both aortic and mitral valve calcification in ESRD.

For all patients and controls the following was done:

1. Full medical history and clinical examination.

2. Laboratory investigations including:

a) Serum creatinine $(\mathrm{mg} / \mathrm{dL})$ : according to the method of Yatzidis (1974).

b) Blood urea $(\mathrm{mg} / \mathrm{dl})$ : according to the method of Tobacco et al., (1979).

c) Serum calcium ( $\mathrm{mg} / \mathrm{dl})$ : according to the method of Renoe et al. (1980).

d) Serum phosphorus (mg/dl): according to the method of Farrel (1987).

e) Serum albumin $(\mathrm{mg} / \mathrm{dl})$ : according to the method of Doumas et al. ( 1971).

f) Parathyroid hormone level ( $\mathrm{ng} / \mathrm{ml})$ : according to the method of Endras et al. (1989).

g) For purposes of the study we calculated the value of calcium phosphorus product $\left(\mathrm{CaxP}\left(\mathrm{mg}^{2} / \mathrm{dl}^{2}\right)\right.$.

\section{Echocardiography:}

According to the standard protocol, a professional skilled cardiologist had performed echocardiographic examination to all patients and control subjects included in the study.

$\mathrm{M}^{-}$mode, two dimensional echocardiography and Doppler ultrasound studies (pulsed, continuous wave and colour flow imaging) were performed using a high resolution (ALT 5000 HDI) scanning device. 
We measured aortic root dimension (AO in $\mathrm{mm})$, valve calcification in ESRD, Right atrium dimension (RA in $\mathrm{mm}$ ), End-diastolic dimensions (EDD in $\mathrm{mm}$ ), End-systolic dimensions (ESD in $\mathrm{mm}$ ), shortening fraction (FS in \%), ejection fraction (EF in \%), interventricular septum dimensions (IVST d in $\mathrm{mm}$ ), and posterior wall dimensions in diastole (PWTd in $\mathrm{mm}$ ).

\section{Statistical methods:}

\section{P-value}

* P-value was considered significant if $<0.05(\mathrm{~S})$.

$*$ P-value was considered borderline significance if $<0.1(\mathrm{BS})$.

* P-value was considered highly significant if $<0.01$ (HS).

$* \mathrm{P}$-value was considered non-significant if $\geq 0.1(\mathrm{NS})$.

Table (1): Frequency distribution of demographic data of group A

\begin{tabular}{|c|c|c|}
\hline Variables & $\mathbf{N}$ & $\%$ \\
\hline $\begin{array}{l}\text { Sex } \\
\qquad \text { Male } \\
\text { Female }\end{array}$ & $\begin{array}{l}36 \\
24\end{array}$ & $\begin{array}{l}60 \\
40\end{array}$ \\
\hline $\begin{array}{l}\text { Primary kidney disease: } \\
\text { Unknown } \\
\text { Diabetes mellitus } \\
\text { Hypertension } \\
\text { Chronic glomerulonephritis }\end{array}$ & $\begin{array}{c}13 \\
12 \\
27 \\
8\end{array}$ & $\begin{array}{c}21.7 \\
20 \\
45 \\
13.3\end{array}$ \\
\hline $\begin{array}{l}\text { Vascular insults: } \\
\text { Non } \\
\text { Peripheral vascular insult } \\
\text { Ischemic heart disease } \\
\text { Stroke }\end{array}$ & $\begin{array}{c}29 \\
19 \\
8 \\
4\end{array}$ & $\begin{array}{c}48.3 \\
31.7 \\
13.3 \\
6.7\end{array}$ \\
\hline $\begin{array}{l}\text { Aortic calcification } \\
\text { Negative } \\
\text { Positive }\end{array}$ & $\begin{array}{l}21 \\
39\end{array}$ & $\begin{array}{l}35 \\
65\end{array}$ \\
\hline $\begin{array}{l}\text { Mitral calcification } \\
\text { Negative } \\
\text { Positive }\end{array}$ & $\begin{array}{l}47 \\
13\end{array}$ & $\begin{array}{l}78.3 \\
21.7\end{array}$ \\
\hline $\begin{array}{l}\text { Aortic regurge } \\
\text { AR } \\
\text { Aortic stenosis } \\
\text { Mitral stenosis }\end{array}$ & $\begin{array}{c}18 \\
0 \\
0\end{array}$ & $\begin{array}{c}10.8 \\
0 \\
0\end{array}$ \\
\hline
\end{tabular}

Table (2): Comparison of age between group A and group B 


\begin{tabular}{|l|c|c|c|c|c|}
\hline \multirow{2}{*}{ Variables } & \multicolumn{2}{|c|}{ Group A } & \multicolumn{2}{c|}{ Group B } & \multirow{2}{*}{ P-value } \\
\cline { 2 - 5 } & Mean & SD & Mean & SD & \\
\hline Age (yrs) & 25.00 & 65.00 & 49.32 & 10.68 & $0.9(\mathrm{NS})$ \\
\hline
\end{tabular}

* Unpaired t-test

Table (3): Comparison of laboratory data between group A and group B

\begin{tabular}{|c|c|c|c|c|c|}
\hline \multirow{2}{*}{ Variables } & \multicolumn{2}{|c|}{ Group A } & \multicolumn{2}{|c|}{ Group B } & \multirow{2}{*}{ P-value } \\
\hline & Mean & SD & Mean & SD & \\
\hline Urea (mg/dl) & 40.00 & 5.00 & 29.24 & 5.03 & $0.0001 *(\mathrm{HS})$ \\
\hline Creatinine (mg/dl) & 0.80 & 0.130 & 1.03 & 0.16 & $0.0001 *(\mathrm{HS})$ \\
\hline Albumin (g/day) & 3.59 & 0.21 & 4.11 & 0.45 & $0.0001 *(\mathrm{HS})$ \\
\hline $\mathrm{Ca}(\mathrm{mg} / \mathrm{dl})$ & 8.47 & 1.07 & 9.22 & 0.47 & $0.0001 *(\mathrm{HS})$ \\
\hline $\mathrm{P}(\mathrm{mg} / \mathrm{dl})$ & 6.26 & 1.80 & 3.43 & 0.56 & $0.0001 *(\mathrm{HS})$ \\
\hline $\mathrm{Ca} \times \mathrm{P}\left(\mathrm{mg}^{2} / \mathrm{dl}^{2}\right)$ & 52.47 & 14.46 & 31.56 & 5.29 & $0.0001 *(\mathrm{HS})$ \\
\hline PTH (mg/dl) & 476.86 & 46.60 & 34.88 & 8.6 & $0.0001 *(\mathrm{HS})$ \\
\hline
\end{tabular}

* Unpaired t-test.

Table (4): Comparison of laboratory data between group A and group B

\begin{tabular}{|l|c|c|c|c|c|}
\hline \multirow{2}{*}{\multicolumn{1}{|c|}{ Variables }} & \multicolumn{2}{c|}{ Group A } & \multicolumn{2}{c|}{ Group B } & \multirow{2}{*}{ P-value } \\
\cline { 2 - 5 } & Mean & SD & Mean & SD & \\
\hline AO $(\mathrm{mm})$ & 21.00 & 4.2 & 29.52 & 4.41 & 0.9 \\
\hline RA $(\mathrm{mm})$ & 27.00 & 6.4 & 35.76 & 3.79 & $0.005^{*}$ \\
\hline EDD $(\mathrm{mm})$ & 35.00 & 68.00 & 50.55 & 7.17 & 0.8 \\
\hline ESD $(\mathrm{mm})$ & 20.00 & 43.00 & 30.77 & 5.21 & $0.007^{*}$ \\
\hline FS $(\%)$ & 29.00 & 54.00 & 39.00 & 5.48 & 0.1 \\
\hline EF $(\%)$ & 55.00 & 84.00 & 68.72 & 6.91 & $0.0001^{*}$ \\
\hline IVSTd $(\mathrm{mm})$ & 7.00 & 1.2 & 8.94 & 1.25 & $0.0001^{*}$ \\
\hline PWTd (mm) & 7.000 & 1.2 & 8.92 & 1.35 & $0.000^{*}$ \\
\hline
\end{tabular}

* Unpaired t-test.

Group 1 constituted 35\% of all patients of group A and it comprised 9 females (42.8\%) and 12 males (57.15\%), group 2 constituted $43.33 \%$ of all patients of group A and it comprised 12 females $(46.15 \%)$ and 14 males $(53.85 \%)$.

Group 3 constituted $21.66 \%$ of all patients of group A and it comprised 3 females $(23.06 \%)$ and 10 males $(76.94 \%)$.

Table (5): Results of valvular calcification and valvular lesions in HD patients

\begin{tabular}{|l|c|c|}
\hline & $\begin{array}{c}\text { Valvular } \\
\text { calcification }\end{array}$ & No valvular calcification \\
\hline Number $(\mathrm{n} \%)$ & $39(65 \%)$ & $21(35 \%)$ \\
\hline Isolated mitral valve calcification & -- & -- \\
\hline Isolated aortic valve calcification & $26(66.66 \%)$ & -- \\
\hline Mitral and aortic valve calcification & $13(33.33 \%)$ & -- \\
\hline Mitral regurge & $17(43.5 \%)$ & -- \\
\hline Mitral stenosis & -- & -- \\
\hline Aortic regurge & $18(46.15 \%)$ & -- \\
\hline Aortic stenosis & -- & - \\
\hline
\end{tabular}

Data are (n \%) 
Table (6): Comparison between hemodialysis patients without valvular calcification (group 1) and with valvular calcification (group 2 and 3).

\begin{tabular}{|l|c|c|c|}
\hline \multicolumn{1}{|c|}{ Variables } & $\begin{array}{c}\text { Group 1 } \\
\text { (n= 21) }\end{array}$ & $\begin{array}{c}\text { Group 2 \& 3 } \\
\text { (n= 39) }\end{array}$ & P-value \\
\hline Age (years) & $38.62 \pm 5.54$ & $54.54 \pm 8.80$ & 0.0001 \\
\hline Duration of hemodialysis (years) & $2.43 \pm 1.35$ & $9.15 \pm 2.83$ & 0.0001 \\
\hline Laboratory data & & & \\
Urea (mg\%) & $132.24 \pm 49.99$ & $111.15 \pm 31.24$ & 0.06 \\
Creatinine (mg\%) & $9.08 \pm 3.35$ & $9.58 \pm 5.67$ & 0.7 \\
Albumin (g\%) & $3.61 \pm 0.16$ & $3.58 \pm 0.23$ & 0.5 \\
Ca (mg\%) & $8.20 \pm 1.00$ & $8.62 \pm 1.09$ & 0.2 \\
P (mg\%) & $4.62 \pm 0.99$ & $7.14 \pm 1.5$ & 0.0001 \\
Cax P (mg /dl $\left.{ }^{2}\right)$ & $37.51 \pm 7.37$ & $60.52 \pm 10.28$ & 0.001 \\
PTH (mg\%) & $596.47 \pm 535.82$ & $412.45 \pm 363.03$ & 0.1 \\
\hline Echocardiographic data & & & \\
AO (mm) & $29.19 \pm 4.30$ & $30.33 \pm 4.5$ & 0.3 \\
LA (mm) & $37.86 \pm 5.99$ & $40.38 \pm 5.76$ & 0.1 \\
EDD (mm) & $50.83 \pm 7.28$ & $49.44 \pm 9.54$ & 0.6 \\
ESD (mm) & $33.60 \pm 6.96$ & $36.99 \pm 9$ & 0.1 \\
FS(\%) & $39.57 \pm 9.50$ & $34.59 \pm 6.92$ & 0.02 \\
EF (\%) & $65.24 \pm 9.28$ & $57.51 \pm 8.97$ & 0.003 \\
IVSTd (mm) & $11.05 \pm 2.16$ & $11.67 \pm 2.07$ & 0.3 \\
PWTd (mm) & $10.95 \pm 2.20$ & $11.83 \pm 1.53$ & 0.08 \\
\hline Vascular disease & & & 0.04 \\
Peripheral vascular disease & $5(23.8 \%)$ & $14(35.9 \%)$ & 0.04 \\
Ischemic heart disease & $1(4.8 \%)$ & $7(17.9 \%)$ & 0.04 \\
Stroke & $0(0 \%)$ & $4(10.3 \%)$ & 0.04 \\
No vascular insults & $15(71.4 \%)$ & $14(35.9 \%)$ & 0.04 \\
\hline Drugs & $1857.14 \pm 654.65$ & $3384.62 \pm 891.93$ & 0.0001 \\
Calcium (mg/day) & $2.93 \pm 0.33$ & $2.31 \pm 0.76$ & 0.001 \\
Alfacalcidol $(\mu \mathrm{g} /$ wk) & & \\
\hline
\end{tabular}

Table (7): Valvular calcification according to the etiology of renal disease

\begin{tabular}{|l|c|}
\hline Etiology of renal disease & $\mathrm{N}=39$ \\
\hline Hypertension & $20(51 \%)$ \\
\hline Diabetes & $10(25.6 \%)$ \\
\hline Chronic glomerulonephritis & $4(10.25 \%)$ \\
\hline Unknown etiology & $5(12.8 \%)$ \\
\hline
\end{tabular}

Table (8): Comparison between group 1 and group 2 as regards demographic data.

\begin{tabular}{|c|c|c|c|c|c|}
\hline \multirow{2}{*}{ Variables } & \multicolumn{2}{|c|}{ Group 1 } & \multicolumn{2}{c|}{ Group 2 } & \multirow{2}{*}{ P-value } \\
\cline { 2 - 5 } & Mean & SD & Mean & SD & 0.0001 (HS) \\
\hline Age (yrs) & 38.62 & 5.54 & 53.73 & 9.46 & 0.0001 (HS) \\
\hline $\begin{array}{c}\text { Duration of primary } \\
\text { kidney disease (yrs) }\end{array}$ & 5.54 & 2.11 & 16.14 & 7.66 & 0.0001 (HS) \\
\hline $\begin{array}{c}\text { Duration of } \\
\text { hemodialysis (yrs) }\end{array}$ & 2.43 & 1.35 & 8.50 & 2.61 & 0.061 \\
\hline
\end{tabular}

\footnotetext{
* Unpaired t-test.
} 
Table (9): Comparison between group 1 and group 2 as regards laboratory data

\begin{tabular}{|c|c|c|c|c|c|}
\hline \multirow{2}{*}{ Variables } & \multicolumn{2}{|c|}{ Group 1} & \multicolumn{2}{|c|}{ Group 2} & \multirow{2}{*}{ P-value } \\
\hline & Mean & SD & Mean & SD & \\
\hline Urea (mg/dl) & 132.24 & 49.99 & 107.88 & 26.88 & $0.04(\mathrm{~S})$ \\
\hline $\begin{array}{c}\text { Creatinine } \\
(\mathrm{mg} / \mathrm{dl})\end{array}$ & 9.08 & 2.35 & 9.89 & 2.74 & $0.6(\mathrm{NS})$ \\
\hline $\begin{array}{c}\text { Albumin } \\
\text { (g/day) }\end{array}$ & 3.61 & 0.16 & 3.59 & 0.24 & $0.7(\mathrm{NS})$ \\
\hline $\mathrm{Ca}(\mathrm{mg} / \mathrm{dl})$ & 8.20 & 1.00 & 8.96 & 0.90 & 0.009 (HS) \\
\hline $\mathrm{P}(\mathrm{mg} / \mathrm{dl})$ & 4.62 & 1.00 & 6.48 & 1.22 & 0.0001 (HS) \\
\hline $\begin{array}{c}\mathrm{Ca} \times \mathrm{P} \\
\left(\mathrm{mg}^{2} / \mathrm{dl}^{2}\right)\end{array}$ & 37.51 & 7.37 & 57.66 & 10.28 & $0.0001(\mathrm{HS})$ \\
\hline PTH (mg/dl) & 596.47 & 135.82 & 362.12 & 85.23 & $0.06(\mathrm{BS})$ \\
\hline
\end{tabular}

* Unpaired t-test.

Table (10): Comparison between group 1 and group 2 as regards echocardiographic parameters

\begin{tabular}{|l|c|c|c|c|c|}
\hline \multirow{2}{*}{\multicolumn{1}{|c|}{ Variables }} & \multicolumn{2}{c|}{ Group 1 } & \multicolumn{2}{c|}{ Group 2 } & \multirow{2}{*}{ P-value } \\
\cline { 2 - 5 } & Mean & SD & Mean & SD & \multirow{2}{*}{$0.6(\mathrm{NS})$} \\
\hline AO $(\mathrm{mm})$ & 29.19 & 4.30 & 29.96 & 4.89 & $0.2(\mathrm{NS})$ \\
\hline RA $(\mathrm{mm})$ & 37.86 & 5.99 & 40.19 & 6.49 & $0.3(\mathrm{NS})$ \\
\hline EDD $(\mathrm{mm})$ & 50.83 & 7.28 & 48.16 & 9.85 & $0.5(\mathrm{NS})$ \\
\hline ESD $(\mathrm{mm})$ & 33.60 & 6.96 & 35.19 & 8.04 & $0.1(\mathrm{NS})$ \\
\hline FS $(\%)$ & 39.57 & 9.50 & 35.38 & 7.68 & $0.02(\mathrm{~S})$ \\
\hline EF $(\%)$ & 65.24 & 9.28 & 58.19 & 9.88 & $0.2(\mathrm{NS})$ \\
\hline IVSTd $(\mathrm{mm})$ & 11.05 & 2.16 & 11.88 & 2.29 & $0.1(\mathrm{NS})$ \\
\hline PWTd $(\mathrm{mm})$ & 10.95 & 2.20 & 11.89 & 1.72 & \multirow{2}{*}{} \\
\hline
\end{tabular}

* Unpaired t-test.

Table (11): Comparison between group 1 and group 2 as regards drug doses.

\begin{tabular}{|c|c|c|c|c|c|}
\hline \multirow{2}{*}{ Variables } & \multicolumn{2}{|c|}{ Group 1} & \multicolumn{2}{|c|}{ Group 2} & \multirow{2}{*}{ P-value } \\
\hline & Mean & SD & Mean & SD & \\
\hline Calcium (mg/dl) & 1857.14 & 454.65 & 3173.08 & 782.52 & $0.04(\mathrm{~S})$ \\
\hline $\begin{array}{l}\text { Alphacalciferol } \\
(\mu \mathrm{g})\end{array}$ & 2.93 & 0.33 & 2.42 & 0.74 & 0.006 (HS) \\
\hline
\end{tabular}

* Unpaired t-test

Table (12): $\quad$ Comparison between group 1 and group 3 as regards demographic data.

\begin{tabular}{|c|c|c|c|c|c|}
\hline \multirow{2}{*}{ Variables } & \multicolumn{2}{|c|}{ Group 1 } & \multicolumn{2}{c|}{ Group 3 } & \multirow{2}{*}{ P-value } \\
\cline { 2 - 5 } & Mean & SD & Mean & SD & \\
\hline Age (yrs) & 38.62 & 5.54 & 56.15 & 7.39 & $0.0001(\mathrm{HS})$ \\
\hline $\begin{array}{c}\text { Duration of primary } \\
\text { kidney disease (yrs) }\end{array}$ & 5.54 & 2.11 & 19.42 & 8.68 & $0.0001(\mathrm{HS})$ \\
\hline $\begin{array}{c}\text { Duration of } \\
\text { hemodialysis (yrs) }\end{array}$ & 2.43 & 1.35 & 10.46 & 2.90 & $0.0001(\mathrm{HS})$ \\
\hline
\end{tabular}

\footnotetext{
* Unpaired t-test.
} 
Table (13): $\quad$ Comparison between group 1 and group 3 as regards laboratory data

\begin{tabular}{|c|c|c|c|c|c|}
\hline \multirow{2}{*}{ Variables } & \multicolumn{2}{|c|}{ Group 1 } & \multicolumn{2}{c|}{ Group 3 } & \multirow{2}{*}{ P-value } \\
\cline { 2 - 5 } & Mean & SD & Mean & SD & \\
\hline Urea $(\mathrm{mg} / \mathrm{dl})$ & 132.24 & 29.99 & 117.69 & 38.93 & $0.4(\mathrm{NS})$ \\
\hline Creatinine $(\mathrm{mg} / \mathrm{dl})$ & 9.08 & 2.35 & 8.96 & 2.56 & $0.9(\mathrm{NS})$ \\
\hline Albumin $(\mathrm{g} / \mathrm{day})$ & 3.61 & 0.16 & 3.56 & 0.21 & $0.4(\mathrm{NS})$ \\
\hline $\mathrm{Ca}(\mathrm{mg} / \mathrm{dl})$ & 8.20 & 1.00 & 7.94 & 1.15 & $0.5(\mathrm{NS})$ \\
\hline $\mathrm{P}(\mathrm{mg} / \mathrm{dl})$ & 4.62 & 1.00 & 8.45 & 1.13 & $0.0001(\mathrm{HS})$ \\
\hline $\mathrm{Cax} \mathrm{P}\left(\mathrm{mg}^{2} / \mathrm{dl}^{2}\right)$ & 37.51 & 7.736 & 66.25 & 7.80 & $0.0001(\mathrm{HS})$ \\
\hline $\mathrm{PTH}(\mathrm{mg} / \mathrm{dl})$ & 596.47 & 53.58 & 513.10 & 48.102 & $0.7(\mathrm{NS})$ \\
\hline
\end{tabular}

* Unpaired t-test.

Table (14): Comparison between group 1 and group 3 as regards ecvhocardiographic parameters

\begin{tabular}{|l|c|c|c|c|c|}
\hline \multirow{2}{*}{ Variables } & \multicolumn{2}{|c|}{ Group 1 } & \multicolumn{2}{c|}{ Group 3 } & \multirow{2}{*}{ P-value } \\
\cline { 2 - 5 } & Mean & SD & Mean & SD & \multirow{2}{*}{$0.2(\mathrm{NS})$} \\
\hline AO $(\mathrm{mm})$ & 29.19 & 4.30 & 31.08 & 3.68 & $0.1(\mathrm{NS})$ \\
\hline RA $(\mathrm{mm})$ & 37.86 & 5.99 & 40.77 & 4.13 & $0.7(\mathrm{NS})$ \\
\hline EDD $(\mathrm{mm})$ & 50.83 & 7.28 & 52.00 & 8.68 & $0.02(\mathrm{~S})$ \\
\hline ESD $(\mathrm{mm})$ & 33.60 & 6.96 & 40.58 & 10.03 & $0.03(\mathrm{~S})$ \\
\hline FS $(\%)$ & 39.57 & 9.50 & 33.00 & 4.95 & $0.005(\mathrm{HS})$ \\
\hline EF $(\%)$ & 65.24 & 9.28 & 56.15 & 6.95 & $0.8(\mathrm{NS})$ \\
\hline IVSTd $(\mathrm{mm})$ & 11.05 & 2.16 & 11.23 & 1.54 & $0.3(\mathrm{NS})$ \\
\hline PWTd $(\mathrm{mm})$ & 10.95 & 2.20 & 11.69 & 1.11 & \\
\hline
\end{tabular}

* Unpaired t-test.

Table (15): $\quad$ Comparison between group 1 and group 3 as regards drug doses

\begin{tabular}{|l|c|c|c|c|c|}
\hline \multirow{2}{*}{ Variables } & \multicolumn{2}{|c|}{ Group 1 } & \multicolumn{2}{c|}{ Group 3 } & \multirow{2}{*}{ P-value } \\
\cline { 2 - 5 } & Mean & SD & Mean & SD & \\
\hline Calcium $(\mathrm{mg})$ & 1857.14 & 454.65 & 3807.69 & 678.31 & $0.0001(\mathrm{HS})$ \\
\hline Alphacalciferol $(\mu \mathrm{g})$ & 2.93 & 0.33 & 2.08 & 0.76 & $0.0001(\mathrm{HS})$ \\
\hline
\end{tabular}

* Unpaired t-test. 
Table (16): Comparison between group 2 and group 3 as regards demographic data

\begin{tabular}{|l|c|c|c|c|c|}
\hline \multirow{2}{*}{ Variables } & \multicolumn{2}{|c|}{ Group 2 } & \multicolumn{2}{c|}{ Group 3 } & \multirow{2}{*}{ P-value } \\
\cline { 2 - 5 } & Mean & SD & Mean & SD & $0.4(\mathrm{NS})$ \\
\hline Age (yrs) & 53.73 & 9.46 & 56.15 & 7.39 & $0.3(\mathrm{NS})$ \\
\hline $\begin{array}{l}\text { Duration of primary kidney } \\
\text { disease (yrs) }\end{array}$ & 16.14 & 7.66 & 19.42 & 8.68 & $0.04(\mathrm{~S})$ \\
\hline $\begin{array}{l}\text { Duration of hemodialysis } \\
\text { (yrs) }\end{array}$ & 8.50 & 2.61 & 10.46 & 2.90 & 0 \\
\hline
\end{tabular}

* Unpaired t-test.

Table (17): Comparison between group 2 and group 3 as regards laboratory data

\begin{tabular}{|l|c|c|c|c|c|}
\hline \multirow{2}{*}{ Variables } & \multicolumn{2}{c|}{ Group 2 } & \multicolumn{2}{c|}{ Group 3 } & \multirow{2}{*}{ P-value } \\
\cline { 2 - 5 } & Mean & SD & Mean & SD & \multirow{2}{*}{$0.4(\mathrm{NS})$} \\
\hline Urea $(\mathrm{mg} / \mathrm{dl})$ & 107.88 & 26.88 & 117.69 & 38.93 & $0.6(\mathrm{NS})$ \\
\hline Creatinine $(\mathrm{mg} / \mathrm{dl})$ & 9.89 & 2.74 & 8.96 & 2.56 & $0.7(\mathrm{NS})$ \\
\hline Albumin $(\mathrm{g} / \mathrm{day})$ & 3.59 & 0.24 & 3.56 & 0.21 & $0.004(\mathrm{HS})$ \\
\hline $\mathrm{Ca}(\mathrm{mg} / \mathrm{dl})$ & 8.96 & 0.90 & 7.94 & 1.15 & $0.0001(\mathrm{HS})$ \\
\hline $\mathrm{P}(\mathrm{mg} / \mathrm{dl})$ & 6.48 & 1.22 & 8.45 & 1.13 & $0.01(\mathrm{HS})$ \\
\hline $\mathrm{Cax}$ P $\left(\mathrm{mg}^{2} / \mathrm{dl}^{2}\right)$ & 57.66 & 10.28 & 66.25 & 7.80 & $0.2(\mathrm{NS})$ \\
\hline PTH $(\mathrm{mg} / \mathrm{dl})$ & 362.12 & 85.23 & 513.10 & 48.102 & \\
\hline
\end{tabular}

* Unpaired t-test.

Table (18): Comparison between group 2 and group 3 as regards echocardiographic parameters

\begin{tabular}{|l|c|c|c|c|c|}
\multirow{2}{*}{\multicolumn{1}{|c|}{ Variables }} & \multicolumn{2}{c|}{ Group A } & \multicolumn{2}{c|}{ Group B } & \multirow{2}{*}{ P-value } \\
\cline { 2 - 5 } & Mean & SD & Mean & SD & \multirow{2}{*}{$0.5(\mathrm{NS})$} \\
\hline AO $(\mathrm{mm})$ & 29.96 & 4.89 & 31.08 & 3.68 & $0.8(\mathrm{NS})$ \\
\hline RA $(\mathrm{mm})$ & 40.19 & 6.49 & 40.77 & 4.13 & $0.2(\mathrm{NS})$ \\
\hline EDD $(\mathrm{mm})$ & 48.16 & 9.85 & 52.00 & 8.68 & $0.1(\mathrm{NS})$ \\
\hline ESD $(\mathrm{mm})$ & 35.19 & 8.04 & 40.58 & 10.03 & $0.3(\mathrm{NS})$ \\
\hline FS $(\%)$ & 35.38 & 7.68 & 33.00 & 4.95 & $0.5(\mathrm{NS})$ \\
\hline EF $(\%)$ & 58.19 & 9.88 & 56.15 & 6.95 & $0.4(\mathrm{NS})$ \\
\hline IVST $(\mathrm{mm})$ & 11.88 & 2.29 & 11.23 & 1.54 & $0.7(\mathrm{NS})$ \\
\hline PW $(\mathrm{mm})$ & 11.89 & 1.72 & 11.69 & 1.11 & \multirow{2}{*}{} \\
\hline
\end{tabular}

* Unpaired t-test.

Table (19): $\quad$ Comparison between group 2 and group 3 as regards drug doses

\begin{tabular}{|l|c|c|c|c|c|}
\hline \multirow{2}{*}{\multicolumn{1}{c|}{ Variables }} & \multicolumn{2}{|c|}{ Group 2 } & \multicolumn{2}{c|}{ Group 3 } & \multirow{2}{*}{ P-value } \\
\cline { 2 - 6 } & Mean & SD & Mean & SD & \\
\hline Calcium $(\mathrm{mg})$ & 3173.08 & 782.52 & 3807.69 & 778.31 & $0.03(\mathrm{~S})$ \\
\hline Alphacalciferol $(\mu \mathrm{g})$ & 2.42 & 0.74 & 2.08 & 0.76 & $0.1(\mathrm{NS})$ \\
\hline
\end{tabular}

\footnotetext{
* Unpaired t-test.
} 
Table (20): Correlation between $\mathrm{Ca}, \mathrm{P}, \mathrm{Cax} \mathrm{P}, \mathrm{PTH}$ and demographic data of patients within group 1.

\begin{tabular}{|l|c|c|c|c|c|}
\hline \multicolumn{1}{|c|}{ Variables } & $\begin{array}{c}\text { Correlation } \\
\text { coefficient }\end{array}$ & Ca & P & CaxP & PTH \\
\hline Age (yrs) & $\mathrm{r}$ & 0.5 & -0.4 & -0.2 & -0.2 \\
& $\mathrm{p}$ & $0.04^{*}(\mathrm{~S})$ & $0.06(\mathrm{BS})$ & $0.5(\mathrm{NS})$ & $0.3(\mathrm{NS})$ \\
\hline $\begin{array}{l}\text { Duration of primary kidney } \\
\text { disease (yrs) }\end{array}$ & $\mathrm{r}$ & 0.4 & -0.256 & -0.1 & 0.2 \\
\hline Duration of hemodialysis (yrs) & $\mathrm{p}$ & $0.2(\mathrm{NS})$ & $0.4(\mathrm{NS})$ & $0.7(\mathrm{NS})$ & $0.5(\mathrm{NS})$ \\
& $\mathrm{p}$ & $0.01 *(\mathrm{HS})$ & $0.6(\mathrm{NS})$ & $0.4(\mathrm{NS})$ & $0.4(\mathrm{NS})$ \\
\hline
\end{tabular}

Pearson Linear Correlation test

Table (21): Correlation between $\mathrm{Ca}, \mathrm{P} \mathrm{CaxP}, \mathrm{PTH}$, and laboratory data of patients within group 1

\begin{tabular}{|c|c|c|c|c|c|}
\hline Variables & $\begin{array}{c}\text { Correlation } \\
\text { coefficient }\end{array}$ & $\mathbf{C a}$ & $\mathbf{P}$ & CaxP & PTH \\
\hline $\mathrm{Ca}(\mathrm{mg} / \mathrm{dl})$ & $\begin{array}{l}\mathrm{r} \\
\mathrm{p}\end{array}$ & & $\begin{array}{c}-0.4 \\
0.05^{*}(\mathrm{~S})\end{array}$ & $\begin{array}{c}0.1 \\
0.6(\mathrm{NS})\end{array}$ & $\begin{array}{c}0.2 \\
0.3(\mathrm{NS})\end{array}$ \\
\hline $\mathrm{P}(\mathrm{mg} / \mathrm{dl})$ & $\begin{array}{l}\mathrm{r} \\
\mathrm{p}\end{array}$ & $\begin{array}{c}-0.4 \\
0.05^{*}(\mathrm{~S})\end{array}$ & & $\begin{array}{c}0.8 \\
0.0001 *(\mathrm{HS})\end{array}$ & $\begin{array}{c}-0.2 \\
0.4(\mathrm{NS})\end{array}$ \\
\hline $\operatorname{Cax} \mathrm{P}\left(\mathrm{mg}^{2} / \mathrm{dl}^{2}\right)$ & $\begin{array}{l}\mathrm{r} \\
\mathrm{p}\end{array}$ & $\begin{array}{c}0.1 \\
0.6(\mathrm{NS})\end{array}$ & $\begin{array}{c}0.8 \\
0.0001 *(\mathrm{HS})\end{array}$ & & $\begin{array}{c}-0.07 \\
0.8(\mathrm{NS})\end{array}$ \\
\hline PTH (mg/dl) & $\begin{array}{l}\mathrm{r} \\
\mathrm{p}\end{array}$ & $\begin{array}{c}0.2 \\
0.3(\mathrm{NS})\end{array}$ & $\begin{array}{c}-0.2 \\
0.4(\mathrm{NS})\end{array}$ & $\begin{array}{c}-0.07 \\
0.8(\mathrm{NS})\end{array}$ & \\
\hline Urea (mg/dl) & $\begin{array}{l}\mathrm{r} \\
\mathrm{p}\end{array}$ & $\begin{array}{c}-0.5 \\
0.02 *(\mathrm{~S})\end{array}$ & $\begin{array}{c}-0.09 \\
0.7(\mathrm{NS})\end{array}$ & $\begin{array}{c}-0.4 \\
0.09(\mathrm{BS})\end{array}$ & $\begin{array}{c}0.3 \\
0.2 \text { (NS) }\end{array}$ \\
\hline Creatinine (mg/dl) & $\begin{array}{l}\mathrm{r} \\
\mathrm{p}\end{array}$ & $\begin{array}{c}-0.5 \\
0.01 *(\mathrm{HS})\end{array}$ & $\begin{array}{c}0.3 \\
0.2(\mathrm{NS})\end{array}$ & $\begin{array}{c}0.005 \\
1.0(\mathrm{NS})\end{array}$ & $\begin{array}{c}0.02 \\
0.9 \text { (NS) }\end{array}$ \\
\hline Albumin (g/day) & $\begin{array}{l}\mathrm{r} \\
\mathrm{p}\end{array}$ & $\begin{array}{c}0.03 \\
0.9(\mathrm{NS})\end{array}$ & $\begin{array}{c}0.1 \\
0.6(\mathrm{NS})\end{array}$ & $\begin{array}{c}0.120 \\
0.6(\mathrm{NS})\end{array}$ & $\begin{array}{c}-0.2 \\
0.3(\mathrm{NS})\end{array}$ \\
\hline
\end{tabular}

Pearson Linear Correlation test 
Table (22): Correlation between $\mathrm{Ca}, \mathrm{P}, \mathrm{CaxP}, \mathrm{PTH}$ and echocardiographic parameters within group 1

\begin{tabular}{|l|c|c|c|c|c|}
\hline \multicolumn{1}{|c|}{ Variables } & $\begin{array}{c}\text { Correlation } \\
\text { coefficient }\end{array}$ & Ca & P & CaxP & PTH \\
\hline AO $(\mathrm{mm})$ & $\mathrm{r}$ & 0.1 & 0.3 & 0.4 & 0.1 \\
& $\mathrm{p}$ & $0.6(\mathrm{NS})$ & $0.2(\mathrm{NS})$ & $0.06(\mathrm{BS})$ & $0.6(\mathrm{NS})$ \\
\hline RA $(\mathrm{mm})$ & $\mathrm{r}$ & 0.1 & 0.04 & 0.1 & -0.3 \\
& $\mathrm{p}$ & $0.7(\mathrm{NS})$ & $0.9(\mathrm{NS})$ & $0.6(\mathrm{NS})$ & $0.2(\mathrm{NS})$ \\
\hline EDD $(\mathrm{mm})$ & $\mathrm{r}$ & 0.02 & -0.2 & -0.3 & 0.3 \\
& $\mathrm{p}$ & $0.9(\mathrm{NS})$ & $0.3(\mathrm{NS})$ & $0.2(\mathrm{NS})$ & $0.3(\mathrm{NS})$ \\
\hline ESD $(\mathrm{mm})$ & $\mathrm{r}$ & 0.2 & 0.2 & 0.3 & 0.08 \\
& $\mathrm{p}$ & $0.5(\mathrm{NS})$ & $0.5(\mathrm{NS})$ & $0.2(\mathrm{NS})$ & $0.7(\mathrm{NS})$ \\
\hline FS $(\%)$ & $\mathrm{r}$ & -0.2 & 0.2 & 0.03 & -0.2 \\
& $\mathrm{p}$ & $0.3(\mathrm{NS})$ & $0.5(\mathrm{NS})$ & $0.9(\mathrm{NS})$ & $0.4(\mathrm{NS})$ \\
\hline EF $(\%)$ & $\mathrm{r}$ & 0.2 & 0.2 & 0.3 & -0.09 \\
\hline IVSTd $(\mathrm{mm})$ & $\mathrm{p}$ & $0.5(\mathrm{NS})$ & $0.4(\mathrm{NS})$ & $0.2(\mathrm{NS})$ & $0.7(\mathrm{NS})$ \\
\hline & $\mathrm{r}$ & 0.2 & 0.1 & 0.3 & -0.09 \\
\hline
\end{tabular}

Pearson Linear Correlation test

Table (23): Correlation between $\mathrm{Ca}, \mathrm{P}, \mathrm{CaxP}, \mathrm{PTH}$ and drug doses within group 1 patients

\begin{tabular}{|l|c|c|c|c|c|}
\hline \multicolumn{1}{|c|}{ Variables } & $\begin{array}{c}\text { Correlation } \\
\text { coefficient }\end{array}$ & Ca & P & CaxP & PTH \\
\hline Calcium $(\mathrm{mg})$ & $\mathrm{r}$ & 0.03 & -0.2 & -0.2 & -0.3 \\
& $\mathrm{p}$ & $0.9(\mathrm{NS})$ & $0.5(\mathrm{NS})$ & $0.5(\mathrm{NS})$ & $0.3(\mathrm{NS})$ \\
\hline Alphacalciferol $(\mu \mathrm{g})$ & $\mathrm{r}$ & -0.2 & 0.2 & 0.09 & 0.08 \\
& $\mathrm{p}$ & $0.4(\mathrm{NS})$ & $0.4(\mathrm{NS})$ & $0.7(\mathrm{NS})$ & $0.7(\mathrm{NS})$ \\
\hline
\end{tabular}

Pearson Linear Correlation test 
Table (24): Correlation between $\mathrm{Ca}, \mathrm{P}, \mathrm{CaxP}, \mathrm{PTH}$ and demographic data within group 2

\begin{tabular}{|l|c|c|c|c|c|}
\hline \multicolumn{1}{|c|}{ Variables } & $\begin{array}{c}\text { Correlation } \\
\text { coefficient }\end{array}$ & Ca & P & CaxP & PTH \\
\hline Age (yrs) & $\mathrm{r}$ & -0.09 & 0.3 & 0.2 & 0.07 \\
\hline $\begin{array}{l}\text { Duration of primary } \\
\text { kidney disease (yrs) }\end{array}$ & $\mathrm{p}$ & $0.6(\mathrm{NS})$ & $0.2(\mathrm{NS})$ & $0.2(\mathrm{NS})$ & $0.7(\mathrm{NS})$ \\
\hline $\begin{array}{l}\text { Duration of } \\
\text { hemodialysis (yrs) }\end{array}$ & $\mathrm{p}$ & $0.4(\mathrm{NS})$ & $0.9(\mathrm{NS})$ & $0.6(\mathrm{NS})$ & $0.3(\mathrm{NS})$ \\
\hline
\end{tabular}

Pearson Linear Correlation test

Table (25): Correlation between Ca, P, CaxP, PTH and laboratory data within group 2

\begin{tabular}{|c|c|c|c|c|c|}
\hline Variables & $\begin{array}{c}\text { Correlation } \\
\text { coefficient }\end{array}$ & $\mathbf{C a}$ & $\mathbf{P}$ & $\operatorname{CaxP}$ & PTH \\
\hline \multirow{2}{*}{$\mathrm{Ca}(\mathrm{mg} / \mathrm{dl})$} & $\mathrm{r}$ & - & -0.4 & 0.2 & -0.4 \\
\hline & $\mathrm{p}$ & - & $0.06(\mathrm{BS})$ & $0.3(\mathrm{NS})$ & 0.9 (NS) \\
\hline \multirow{2}{*}{$\mathrm{P}(\mathrm{mg} / \mathrm{dl})$} & $\mathrm{r}$ & -0.4 & - & 0.8 & -0.05 \\
\hline & $\mathrm{p}$ & $0.06(\mathrm{BS})$ & - & $0.0001 *(\mathrm{HS})$ & $0.8(\mathrm{NS})$ \\
\hline \multirow{2}{*}{$\operatorname{Cax} \mathrm{P}\left(\mathrm{mg}^{2} / \mathrm{dl}^{2}\right)$} & $\mathrm{r}$ & 0.2 & 0.8 & - & -0.05 \\
\hline & $\mathrm{p}$ & $0.3(\mathrm{NS})$ & $0.0001 *(\mathrm{HS})$ & - & $0.8(\mathrm{NS})$ \\
\hline \multirow{2}{*}{ PTH (mg/dl) } & $\mathrm{r}$ & -0.04 & -0.05 & -0.05 & - \\
\hline & $\mathrm{p}$ & $0.9(\mathrm{NS})$ & $0.8(\mathrm{NS})$ & $0.8(\mathrm{NS})$ & - \\
\hline \multirow{2}{*}{ Urea (mg/dl) } & $\mathrm{r}$ & -0.2 & 0.1 & -0.008 & -0.2 \\
\hline & $\mathrm{p}$ & $0.2(\mathrm{NS})$ & $0.6(\mathrm{NS})$ & $0.6(\mathrm{NS})$ & $0.3(\mathrm{NS})$ \\
\hline \multirow{2}{*}{ Creatinine (mg/dl) } & $\mathrm{r}$ & 0.05 & 0.1 & 0.2 & 0.05 \\
\hline & $\mathrm{p}$ & $0.8(\mathrm{NS})$ & $0.6(\mathrm{NS})$ & $0.4(\mathrm{NS})$ & 0.8 (NS) \\
\hline \multirow{2}{*}{ Albumin (g/day) } & $\mathrm{r}$ & 0.3 & 0.04 & 0.2 & 0.08 \\
\hline & $\mathrm{p}$ & $0.2(\mathrm{NS})$ & $0.8(\mathrm{NS})$ & 0.3 (NS) & $0.7(\mathrm{NS})$ \\
\hline
\end{tabular}

Pearson Linear Correlation test 
Table (26): Correlation between $\mathrm{Ca}, \mathrm{P}, \mathrm{CaxP}, \mathrm{PTH}$ and echocardiographic parameters within group 2

\begin{tabular}{|c|c|c|c|c|c|}
\hline Variables & $\begin{array}{c}\text { Correlation } \\
\text { coefficient }\end{array}$ & $\mathbf{C a}$ & $\mathbf{P}$ & CaxP & PTH \\
\hline \multirow{2}{*}{$\mathrm{AO}(\mathrm{mm})$} & $\mathrm{r}$ & -0.07 & 0.2 & 0.2 & 0.04 \\
\hline & $\mathrm{p}$ & $0.7(\mathrm{NS})$ & 0.3 (NS) & 0.4 (NS) & 0.9 (NS) \\
\hline \multirow{2}{*}{$\mathrm{RA}(\mathrm{mm})$} & $\mathrm{r}$ & 0.2 & -0.3 & -0.2 & 0.3 \\
\hline & $\mathrm{p}$ & $0.3(\mathrm{NS})$ & $0.1(\mathrm{NS})$ & $0.2(\mathrm{NS})$ & 0.1 (NS) \\
\hline \multirow{2}{*}{$\mathrm{EDD}(\mathrm{mm})$} & $\mathrm{r}$ & 0.02 & -0.2 & -0.2 & 0.07 \\
\hline & $\mathrm{p}$ & $0.9(\mathrm{NS})$ & $0.4(\mathrm{NS})$ & $0.4(\mathrm{NS})$ & 0.7 (NS) \\
\hline \multirow{2}{*}{ ESD (mm) } & $\mathrm{r}$ & -0.06 & -0.02 & -0.07 & -0.02 \\
\hline & $\mathrm{p}$ & $0.8(\mathrm{NS})$ & $0.9(\mathrm{NS})$ & $0.7(\mathrm{NS})$ & 0.9 (NS) \\
\hline \multirow{2}{*}{ FS (\%) } & $\mathrm{r}$ & 0.01 & 0.4 & 0.5 & 0.05 \\
\hline & $\mathrm{p}$ & $0.9(\mathrm{NS})$ & $0.03 *(\mathrm{~S})$ & $0.01 *(\mathrm{HS})$ & 0.8 (NS) \\
\hline \multirow{2}{*}{$\mathrm{EF}(\%)$} & $\mathrm{r}$ & -0.4 & 0.08 & -0.1 & -0.4 \\
\hline & $\mathrm{p}$ & $0.06(\mathrm{BS})$ & $0.7(\mathrm{NS})$ & $0.5(\mathrm{NS})$ & $0.02(\mathrm{~S})$ \\
\hline \multirow{2}{*}{ IVSTd (mm) } & $\mathrm{r}$ & 0.04 & 0.02 & 0.05 & -0.01 \\
\hline & $\mathrm{p}$ & $0.8(\mathrm{NS})$ & $0.9(\mathrm{NS})$ & $0.7(\mathrm{NS})$ & 0.9 (NS) \\
\hline \multirow{2}{*}{ PWTd (mm) } & $\mathrm{r}$ & 0.1 & 0.08 & 0.2 & 0.06 \\
\hline & $\mathrm{p}$ & $0.6(\mathrm{NS})$ & $0.7(\mathrm{NS})$ & $0.4(\mathrm{NS})$ & 0.8 (NS) \\
\hline
\end{tabular}

Pearson Linear Correlation test

Table (27): $\quad$ Correlation between Ca, P, Cax P, PTH and drug doses within group 2

\begin{tabular}{|l|c|c|c|c|c|}
\hline \multicolumn{1}{|c|}{ Variables } & $\begin{array}{c}\text { Correlation } \\
\text { coefficient }\end{array}$ & Ca & P & CaxP & PTH \\
\hline Calcium $(\mathrm{mg})$ & $\mathrm{r}$ & 0.4 & -0.4 & -0.2 & -0.08 \\
& $\mathrm{p}$ & $0.04(\mathrm{~S})$ & $0.06(\mathrm{BS})$ & $0.5(\mathrm{NS})$ & $0.7(\mathrm{NS})$ \\
\hline \multirow{2}{*}{ Alphacalciferol $(\mu \mathrm{g})$} & $\mathrm{r}$ & -0.4 & 0.3 & 0.1 & 0.2 \\
& $\mathrm{p}$ & $0.03(\mathrm{~S})$ & $0.1(\mathrm{NS})$ & $0.6(\mathrm{NS})$ & $0.3(\mathrm{NS})$ \\
\hline
\end{tabular}

Pearson Linear Correlation test 
Table (28): Correlation between $\mathrm{Ca}, \mathrm{P}, \mathrm{CaxP}, \mathrm{PTH}$ and demographic data within group 3

\begin{tabular}{|l|c|c|c|c|c|}
\hline \multicolumn{1}{|c|}{ Variables } & $\begin{array}{c}\text { Correlation } \\
\text { coefficient }\end{array}$ & Ca & P & CaxP & PTH \\
\hline Age (yrs) & $\mathrm{r}$ & 0.09 & -0.4 & -0.3 & -0.6 \\
& $\mathrm{p}$ & $0.7(\mathrm{NS})$ & $0.2(\mathrm{NS})$ & $0.4(\mathrm{NS})$ & $0.05 *(\mathrm{~S})$ \\
\hline $\begin{array}{l}\text { Duration of primary } \\
\text { kidney disease (yrs) }\end{array}$ & $\mathrm{r}$ & -0.1 & -0.3 & -0.5 & 0.01 \\
\hline $\begin{array}{l}\text { Duration of } \\
\text { hemodialysis (yrs) }\end{array}$ & $\mathrm{p}$ & $0.7(\mathrm{NS})$ & $0.4(\mathrm{NS})$ & $0.1(\mathrm{NS})$ & $0.9(\mathrm{NS})$ \\
\hline
\end{tabular}

Pearson Linear Correlation test

Table (29): Correlation between $\mathrm{Ca}, \mathrm{P}, \mathrm{CaxP}, \mathrm{PTH}$ and laboratory data within group 3

\begin{tabular}{|l|c|c|c|c|c|}
\hline \multicolumn{1}{|c|}{ Variables } & $\begin{array}{c}\text { Correlation } \\
\text { coefficient }\end{array}$ & Ca & P & CaxP & PTH \\
\hline $\mathrm{Ca}(\mathrm{mg} / \mathrm{dl})$ & $\mathrm{r}$ & - & -0.7 & 0.5 & -0.3 \\
& $\mathrm{p}$ & - & $0.01 *(\mathrm{HS})$ & $0.06(\mathrm{BS})$ & $0.2(\mathrm{NS})$ \\
\hline $\mathrm{P}(\mathrm{mg} / \mathrm{dl})$ & $\mathrm{r}$ & -0.7 & - & 0.3 & 0.3 \\
\hline & $\mathrm{p}$ & $0.01 *(\mathrm{HS})$ & - & $0.4(\mathrm{NS})$ & $0.4(\mathrm{NS})$ \\
\hline $\left.\mathrm{Cax} \mathrm{P}(\mathrm{mg} / \mathrm{dl})^{2}\right)$ & $\mathrm{r}$ & 0.5 & 0.3 & - & -0.1 \\
\hline PTH $(\mathrm{mg} / \mathrm{dl})$ & $\mathrm{p}$ & $0.06(\mathrm{BS})$ & $0.4(\mathrm{NS})$ & - & $0.7(\mathrm{NS})$ \\
\hline Urea $(\mathrm{mg} / \mathrm{dl})$ & $\mathrm{r}$ & -0.3 & 0.3 & -0.1 & - \\
& $\mathrm{p}$ & $0.2(\mathrm{NS})$ & $0.4(\mathrm{NS})$ & $0.7(\mathrm{NS})$ & - \\
\hline Albumin $(\mathrm{g} / \mathrm{day})$ & $\mathrm{r}$ & -0.1 & 0.03 & -0.1 & 0.7 \\
\hline & $\mathrm{p}$ & $0.6(\mathrm{NS})$ & $0.9(\mathrm{NS})$ & $0.6(\mathrm{NS})$ & $0.01 *(\mathrm{HS})$ \\
\hline
\end{tabular}

Pearson Linear Correlation test 
Table (30): Correlation between $\mathrm{Ca}, \mathrm{P}, \mathrm{CaxP}, \mathrm{PTH}$ and echocardiographic data within group 3, Pearson Linear Correlation test

\begin{tabular}{|c|c|c|c|c|c|}
\hline Variables & $\begin{array}{c}\text { Correlation } \\
\text { coefficient }\end{array}$ & $\mathbf{C a}$ & $\mathbf{P}$ & CaxP & PTH \\
\hline \multirow{2}{*}{$\mathrm{AO}(\mathrm{mm})$} & $\mathrm{r}$ & -0.09 & 0.04 & -0.07 & 0.4 \\
\hline & $\mathrm{p}$ & 0.8 (NS) & $0.9(\mathrm{NS})$ & 0.8 (NS) & $0.2(\mathrm{NS})$ \\
\hline \multirow{2}{*}{$\mathrm{RA}(\mathrm{mm})$} & $\mathrm{r}$ & 0.4 & -0.2 & 0.3 & 0.2 \\
\hline & $\mathrm{p}$ & $0.2(\mathrm{NS})$ & $0.5(\mathrm{NS})$ & $0.4(\mathrm{NS})$ & $0.5(\mathrm{NS})$ \\
\hline \multirow{2}{*}{$\mathrm{EDD}(\mathrm{mm})$} & $\mathrm{r}$ & 0.2 & 0.2 & 0.4 & 0.3 \\
\hline & $\mathrm{p}$ & $0.6(\mathrm{NS})$ & $0.6(\mathrm{NS})$ & $0.2(\mathrm{NS})$ & $0.3(\mathrm{NS})$ \\
\hline \multirow{2}{*}{$\mathrm{ESD}(\mathrm{mm})$} & $\mathrm{r}$ & 2 & -0.4 & -0.2 & -0.1 \\
\hline & $\mathrm{p}$ & $0.5(\mathrm{NS})$ & $0.2(\mathrm{NS})$ & $0.6(\mathrm{NS})$ & $0.89 \mathrm{NS})$ \\
\hline \multirow{2}{*}{$\mathrm{FS}(\%)$} & $\mathrm{r}$ & 0.01 & -0.01 & -0.04 & 0.04 \\
\hline & $\mathrm{p}$ & 0.9 (NS) & 0.9 (NS) & $0.9(\mathrm{NS})$ & 0.9 (NS) \\
\hline \multirow{2}{*}{$\mathrm{EF}(\%)$} & $\mathrm{r}$ & 0.08 & 0.1 & 0.2 & -0.2 \\
\hline & $\mathrm{p}$ & $0.8(\mathrm{NS})$ & $0.6(\mathrm{NS})$ & $0.5(\mathrm{NS})$ & $0.4(\mathrm{NS})$ \\
\hline \multirow{2}{*}{ IVSTd (mm) } & $\mathrm{r}$ & -0.5 & -0.03 & -0.6 & 09 \\
\hline & $\mathrm{p}$ & $0.1(\mathrm{NS})$ & $0.9(\mathrm{NS})$ & $0.03 *(\mathrm{~S})$ & $0.7(\mathrm{NS})$ \\
\hline \multirow{2}{*}{ PWTd (mm) } & $\mathrm{r}$ & -0.04 & -0.5 & -0.6 & -0.07 \\
\hline & $\mathrm{p}$ & $0.9(\mathrm{NS})$ & $0.08(\mathrm{BS})$ & $0.03 *(\mathrm{~S})$ & $0.8(\mathrm{NS})$ \\
\hline
\end{tabular}

Table (31): $\quad$ Correlation between $\mathrm{Ca}, \mathrm{P}, \mathrm{CaxP}, \mathrm{PTH}$ and drug doses within group 3 Pearson Linear Correlation test

\begin{tabular}{|l|c|c|c|c|c|}
\hline \multicolumn{1}{|c|}{ Variables } & Correlation coefficient & Ca & P & CaxP & PTH \\
\hline \multirow{2}{*}{ Calcium $(\mathrm{mg})$} & $\mathrm{r}$ & -0.3 & 0.6 & 0.2 & 0.2 \\
& $\mathrm{p}$ & $0.2(\mathrm{NS})$ & $0.05(\mathrm{~S})$ & $0.5(\mathrm{NS})$ & $0.6(\mathrm{NS})$ \\
\hline \multirow{2}{*}{ Alphacalciferol $(\mu \mathrm{g})$} & $\mathrm{r}$ & -0.3 & 3 & -0.06 & 0.7 \\
& $\mathrm{p}$ & $0.3(\mathrm{NS})$ & $0.4(\mathrm{NS})$ & $0.9(\mathrm{NS})$ & $0.007 *(\mathrm{HS})$ \\
\hline
\end{tabular}

Table (32): Predictors of valvular calcification in multiple linear regression analysis in hemodialysis patients.

\begin{tabular}{|l|c|c|c|}
\hline \multicolumn{1}{|c|}{ Variables } & $\mathbf{B}^{*}$ & $\begin{array}{c}\text { 95\% confidence } \\
\text { interval }\end{array}$ & P-value \\
\hline Constant & -0.1 & $-0.7-0.5$ & 0.7 \\
\hline Duration of hemodialysis (yrs) & 0.01 & $0.02-0.07$ & 0.0001 \\
\hline CaxP $\left(\mathrm{mg}^{2} / \mathrm{dl}^{2}\right)$ & 0.01 & $0.004-0.02$ & 0.002 \\
\hline EF $(\%)$ & -0.001 & $-0.02-0.004$ & 0.003 \\
\hline Age of patients (years) & 0.01 & $0.002-0.02$ & 0.01 \\
\hline
\end{tabular}

$\mathrm{R} 2=0.78, \mathrm{SE}($ standard error $)=0.22{ }^{*} \mathrm{~B}($ standard regression coefficient $)$ 
- A multiple stepwise linear regression analysis was performed to determine the independent factors of valvular calcification in hemodialysis patients. Variables significantly associated with valvular calcification and other known to have effect on valvular calcification were introduced in the model. After adjustment for parameters found significant in the univariate analysis, the independent predictors of valvular calcification were duration of hemodialysis, CaxP product, age and reduced EF\%.

- We didn't find any significant correlation between Ca, P, CaxP, PTH and age of healthy controls within group B.

- We didn't find any significant correlation between Ca, P, CaxP, PTH and laboratory data within group B.

- We didn't find significant correlations between Ca, P, CaxP, PTH and echocardiographic parameters within group B.

\section{DISCUSSION}

Lesions of cardiovascular calcification are also seen in dialysis patients at a much younger age than in the general population. Even adults less than 30 years of age on dialysis have a high incidence of coronary artery calcification and these lesions progress at a relatively rapid rate (Isidro \& William, 2002).

Calcification of the mitral and aortic valves. Valvular calcification is a marker of systemic cardiovascular disease in CKD-stage 5 patients (Raggi et al., 2011).

Echocardiography is the gold standard for assessment of cardiac valve morphology and function. It is non invasive and relatively inexpensive. Calcification of the cardiac valves is found in dialysis patients with a prevalence four to five times higher than in the general population (Straumann et al., 1992; Mazzaferro et al., 1993 and Ribeiro et al., 1998).

In our present study on 60 patients on regular $\mathrm{HD}$, of age $($ mean $\pm \mathrm{SD}=$
49.32 \pm 10.68 ) years, the frequency of valvular calcification was 39 patients, 26 patients $(43.33 \%)$ with aortic calcification and 13 patients (21.66\%) with both aortic and mitral calcification. None of the patients had mitral calcification only. Also non of our patients had pulmonary or tricuspid valve pathology. The presence of valvular calcifications in ESRD patients is remarkably high in all published series. Braum et al. (1996), initially studied 49 maintenance HD patients, and identified calcification of mitral valve in $59 \%$ of case and aortic valve in 55\%. Ribeiro et al. (1998) found among 92 patients whose mean age was 60 years, $44 \%$ had mitral valve calcifications on echocardiography, and $52 \%$ had aortic valve calcifications. Nearly $60 \%$ of these patients demonstrated calcifications of both valves. In other studies, mitral valve calcifications were reported in $45 \%$, and aortic valve calcifications in $34 \%$ of patients, while 
both valves were affected in $21 \%$ of the patients studied (Raggi et al., 2002). Valvular thickening and sclerosis, is a frequent finding in HD patients. Among such patients, aortic and mitral valve sclerosis occur in 55 to 69 and 40 to 60 percent of individuals, respectively (Stinebaugh et al., 1995 and Straumann et al., 1992).

In the Jackson cohort of the atherosclerotic risk in communities study (Fox et al., 2004), the Framingham offspring study (Fox et al., 2006), and the Multi-ethnic study of atherosclerosis (MESA) (Ix et al., 2007) cross sectional associations were seen between CKD and mitral annular calcification. In addition, the cardiovascular health study (CHS) showed a high prevalence of CKD in adults with combined mitral annular calcification, aortic annular calcification, and aortic valvular stenosis (Barash et al., 2006).

Non of our control group had aortic or mitral valve calcification. In our study patients with no calcification ( group 1 )had a relatively short hemodialysis duration $($ mean $\pm \mathrm{SD}=2.43 \pm 1.35)$ years, compared to patients with calcified aortic valve ( group 2 ) who had a duration on $\mathrm{HD}($ meran $\pm \mathrm{SD}=8.5 \pm 2.61)$ years with a highly statistically significant difference between both $(\mathrm{p}=0.0001)$. Patients with calcified aortic and mitral valve ( group 3 ) had a hemodialysis duration of (mean \pm SD $=10.46 \pm 2.90)$ years with a highly significant statistical difference between group 3 and group $1(\mathrm{p}=0.0001)$ and a statistically significant difference between group 3 and group $2(\mathrm{p}=0.04)$. This means that hemodialysis duration is implicated in the occurrence of cardiac calcification and also implicated in the extent of calcification. Patients with valvular calcification receiving hemodialysis, had longer hemodialysis duration (Vokov et al., 2009

and Strozecki et al., 2005), echocardiographically examined $65 \mathrm{HD}$ patients ages $(49 \pm 12)$. Years, with duration of HD therapy $(38 \pm 32)$ months. Valvular calcification were found in 32 of 65 patients (49\%), mitral valve calcifications in 10, aortic valve calcifications in 9, and both valves calcifications in 13 patients. In patients with valvular calcification, duration of HD therapy was longer. Also Ribeiro et al. (1998), study suggests that patients with valvular calcification were on long-term renal replacement therapy.

In our study, age of patients with no calcification was $($ mean $\pm \mathrm{SD}=38.62 \pm 5.54)$ years, while age of patients with calcified aortic valve was $($ mean $\pm \mathrm{SD}=$ $853.73 \pm 9.46)$ years. Age of patients with calcified aortic and mitral valve was $($ mean \pm SD $=56.15 \pm 7.39)$ years. Patients with calcified aortic valve showed a statistically higher age when compared to patients with no calcification $(\mathrm{p}=0.0001)$. The same result was found on comparing parients with calcified aortic and mitral valve and patients with no calcification ( $\mathrm{p}$ 
$=0.001)$. While patients with calcified aortic valve and patients with calcified aortic and mitral calcification didn't show significant difference as regards age $(\mathrm{p}=$ $0.4)$. This agrees with studies that suggest that valvular calcification is common in old age patients, group B and group A didn't show significant difference as regards age ( $\mathrm{p} 0.9)$.

Ribeiro et al. (1998) reported that mitral valve calcification was associated with age. Ikee et al. (2010) suggested that mitral valve calcification was associated with increased age.

Ribeiro et al. (1998) reported that aortic valve calcification was correlated with age. Ikee et al. (2010), detected heart valve calcification using two dimensional echocardiography in patients treated with HD three times a week. In 112 patients, (77 men and 35 women, age $(67 \pm 10)$ years, duration of HD $(95 \pm 67)$ months, aortic and mitral valvular calcification were observed in $84(75.0 \%)$ and 58 (51.7\%) patients, respectively aortic valve calcification was associated with age.

Aortic valve calcification is found in 25 to 55 percent of HD patients occurring 10 to 20 years earlier than in the general population (Nasri et al., 2004)

Mitral annular calcification occurs earlier in patients with chronic renal failure than those without renal dysfunction (Forman et al., 1984).

In our study, 12 patients (20\%) of group A were diabetic, 10 patients had valvular calcification.
Rufino et al. (2003), had a cross sectional observational study, patients with valvular calcification were more commonly diabetic.

In our study, 27 patients (4\%) of group A, were hypertensive, 20 patients had valvular calcifications, all of them had a history of predialysis hypertension.

In our study we found 20 patients with aortic calcification who had predialysis hypertension, also patients with aortic insufficiency who suffered from diabetes were 4 patients while patients with aortic insufficiency who suffered from hypertension were 9 out of a total of 15 patients with aortic insufficiency.

In Ribeiro et al. (1998) study patients with valvular calcification had longer duration of predialysis arterial hypertension. Previous studies have reported a history of hypertension prior to starting dialysis as a predictor of valvular calcifications (Braun et al., 1996; Ribeiro et al., 1998 and Yoshida et al., 1999). Braun et al. (1996), studied 49 chronic HD patients aged 28 to 74 years that were compared to 102 nondialysis patients aged 32 to 73 years. They assessed calcification of mitral and aortic valves. The mitral valve was calcified in $59 \%$ of dialysis patients, while aortic valve was calcified in55\%. They drew attention to hypertension as an important risk factor in this process.

Huting et al. (1994) observed that mitral valvular calcifications was associated with the severity of predialysis hypertension. 
Ribeiro et al. (1998) study suggests no correlation was found between valvular calcifications and arterial hypertension, but he reported that aortic valvular calcification was related with duration of hypertension and with the longer duration of predialysis arterial hypertension.

Aortic insufficiency following aortic valve calcification may be due to the progression of the complications of hypertension (Kahnooj et al., 2010).

Urea level was significantly higher in patients with no calcification as compared to patients with calcified aortic valve ( $p$ 0.04 ), this is goes with what was expected that uremia is one the risk factors for calcification.

Low serum albumin is not specific for valvular calcification groups (2 and 3), but also it does affect (group 1) without calcification, seeming to be a characteristic of ESRD and HD.

In contrast to our study, Ikee et al. (2010) stated that aortic valve calcification was associated with lower serum albumin. $\mathrm{Ca}$, $\mathrm{P}$, and CaxP were highly significantly lower in group $\mathrm{A}$ than group $\mathrm{B}(\mathrm{p}=$ 0.0001) but PTH was much higher in group A ( $\mathrm{p}=0.0001), \mathrm{Ca}, \mathrm{P}, \mathrm{CaxP}$, were significantly lower in group 1with no calcification as compared to group 2 (with aortic calcification) $(\mathrm{p}=0.009,0.0001$, 0.0001) respectively. PTH was significantly higher in group 1 than group $2(\mathrm{p}=0.06)$, which is against what was expected.
$\mathrm{P}$ and $\mathrm{C}$ ax $\mathrm{P}$ product were higher in patients with calcified aortic and mitral valve as compared to patients with no calcification $(\mathrm{p}=0.001)$, which points to the role played by $\mathrm{P}$ and $\mathrm{CaxP}$ product in calcification. At same time, PTh didn't show significant difference between the two groups, which means that it has a less important role in valvular calcification (being insignificantly higher in group $1, \mathrm{p}$ $=0.7$ ).

Hyperphosphatemia may directly cause or exacerbate other aspects of CKD-MBD, including secondary hyperparathyroidism, decreased serum calcitriol levels, abnormal bone remodeling and soft-tissue calcification (Katrin et al., 2010).

$\mathrm{Ca}$ is significantly higher in patients with calcified aortic valve than in patients with calcified aortic and mitral valve $(\mathrm{p}=$ 0.004). $\mathrm{P}$ and CaxP product were much higher in patients with calcified aortic and mitral valve than in patients with aortic calcification, again incriminating them of the more extensive calcification of the heart valves.

PTH was insignificantly higher in patients with calcified aortic and mitral valve than in patients with calcified aortic valve $(\mathrm{p}=$ $0.2)$.

Persistent elevations in serum calcium and phosphorus levels can be aggravated by the large doses of vitamin D sterols used often to treat secondary hyperparathyroidism (Raggi et al., 2011).

Tarras et al. (2006) studied 90 patients on maintenance HD for more than 12 months, 
$36(40 \%)$ presented with valvular calcifications and showed higher levels of serum calcium $\quad(92.00 \pm 7.54 \quad$ versus 89.27 $\pm 6.86 \mathrm{mg} / \mathrm{L}, \mathrm{p}=0.04)$, phosphorus $(69.70 \pm 18.33$ versus $44.90 \pm 12.43 \mathrm{mg} / \mathrm{L},(\mathrm{p}$ $<0.001)$, CaxP product $(6164.97 \pm 1797.64$ versus $4024.70 \pm 1066.40 \mathrm{mg}^{2} / \mathrm{L}^{2}, \quad(\mathrm{P}<$ 0.0001 ) as compared with patients without valvular calcifications.

Volkov et al. (2009), reported that patients with valvular calcification had high serum calcium level. This was not the case in our study as regards serum calcium.

Strozeeki et al. (2005) study showed that no statistical significant differences were found with respect to $\mathrm{Ca}, \mathrm{P}, \mathrm{PTH}$ and mean CaxP product in patients with or without valvular calcifications, but the incidence of CaxP product above 44.3 $\mathrm{mmol}^{2} / \mathrm{L}^{2}$ was higher in valvular calcifications compared with those without valvular calcifications.

Aortic valve calcification was associated with higher serum calcium. Mitral valve calcification was not associated with high serum calcium as stated by Ikee et al.(2010).

Torun et al. (2005), found that significantly higher phosphate level was observed in patients with valvular calcifications $(5.1 \pm 1.4$ versus $4.5 \pm 1.4 \mathrm{mg} / \mathrm{dL}, \mathrm{p}=0.04)$. Rufino et al. (2003) had a cross sectional observational study of a cohort of 52 stable patients on maintenance HD for more than 12 months. 20 patients $(38.4 \%)$ presented with valvular calcifications and showed higher levels of serum phosphorus compared to patients without valvular calcifications.

In the presence of low calcium, high phosphours, vitamin D deficiency and uremia parathyroid cells leave quiescence (Cozzolino et al., 2009).

Small decreases in serum $\mathrm{Ca}(++)$ and more prolonged increases in phosphate stimulate the parathyroid to secrete parathyroid hormone (Rochelle et al., 2010).

Calcium can drive the initial steps in hydroxyapatite formation and small changes in calcium concentration have profound effects on calcification of aorta(O'Neill et al., 2011).

Torum et al. (2005), found that CaxP product was high in the study population (48.6 \pm 16.2 versus $39.8 \pm 11.8, \mathrm{p}=0.01)$. Rufino et al. (2003) illustrated that a CaxP product level > $43 \mathrm{mg}^{2} / \mathrm{dL}^{2}$ was the optimal value in terms of sensitivity and specificity for predicting the presence of valvular calcification in their patients population.

Multiple stepwise regression analysis selected CaxP as one of the most predictive parameters for mitral calcification (Ribeiro et al., 1998).

Kahnooj et al. (2010), findings showed that the optimal cut-off point for the CaxP. Product measurement for predicting the severity of aortic valve calcification was $42 \mathrm{mg}^{2} / \mathrm{dL}^{2}$. This discrimination level was different in other studies. Movilli et al. (2005), obtained a break point of 55 
$\mathrm{mg}^{2} / \mathrm{dL}^{2}$ for an optimal CaxP product discrimination value.

Volkov et al. (2009), stated that patients with valvular calcification had high blood parathyroid hormone level while Ribeiro et al. (1998) found no correlation between valvular calcification and parathyroid hormone level. Relatively low PTH concentrations have been associated with a high prevalence of soft tissue and vascular calcification among patients receiving dialysis (Raggi et al., 2011).

Patients with no calcification showed a higher significant doses of calcium and alphacalcidiol than patients with calfied aortic valve ( $p=0.04,0.006)$ respectively, this is against what was expected. $\mathrm{Ca}$ doses was much higher in patients with calcified aortic and mitral valve than patients with no calcification $(\mathrm{p}=0.0001)$ but alphacalcidiol was significantly lower in patients with calcified aortic and mitral valve and patients with no calcification ( $\mathrm{p}$ $=0.0001)$.

Patients with calcified aortic and mitral valve had a higher significant doses of calcium compared to patients with calcified aortic valve ( $\mathrm{p}=0.03)$, but alphacalcidiol was non-significantly higher in patients with calcified aortic valve than in patients with calcified aortic and mitral valve $(P=0.1)$. Does this point to calcium drug as a calcification progression factor whenever calcification occurs. On the contrary does alfacalciferol protect patients with and without calcification from progression of calcification by its well known antiinflammatory effect over body tissues.

Effects of vitamin D such as modulation of an inflammatory response may have a preventive effect (Razzaque, 2011).

Increasing evidence suggests that calcium supplementation may enhance soft tissue calcification and cardiovascular disease in CKD-MBD (Peacock, 2010). The thrombotic or ulcerative changes that occur within heavily calcified valves when exposed to the intracardiac lumen can result in an erupted mass of calcium entering circulation.

Numerous studies have shown that in animal models of uremia, vascular calcification appears to be accelerated by the administration of vitamin D (Mizobuchi et al., 2007).

Tarras et al. (2006) studied 90 patients on maintenance HD for more than 12 months, 36 patients $(60 \%)$ presented with valvular calcification patients with valvular calcification required higher doses of alfacalcidiol for treating secondary hyperparathyroidism $\quad(0.43 \pm 0.60 \quad$ versus $0.11 \pm 0.46 \mu /$ day, $\mathrm{p}<0.0001$ ).

This was not the case in our study.

On making correlations within group 1( patients with no calcification), we found a positive correlation between age and calcium ( $\mathrm{p}=0.04$ ) which could explain calcification of elderly valves in ESRD patients on regular HD. We found borderline significant negative correlation between phosphorus and age $(\mathrm{P}=0.06)$. Also calcium had a highly significant positive correlation 
with duration of dialysis . Calcium and phosphorus had a negative correlation which is well known. A positive correlation was found between $\mathrm{P}$ and CaxP which supports our study opinion of incriminating both in the process of extraosseous calcification in ESRD on HD, we found a negative correlation of calcium with creatinine and urea which is well known.

In group 2 (aortic valve calcification), we found a negative highly significant correlation between serum calcium and duration of dialysis which is against incriminating serum calcium in valvular calcification process in ESRD on HD.

Serum ionized calcium concentration is maintained in a very limited range thanks to parathyroid hormone (PTH) and the active vitamin D metabolite calcitriol. A decreas in ionized calcium level inactivates the calcium sensoring receptor (CaSR), a membranous protein located principally in the parathyroid glands and the kidney, thus stimulating PTH secretion

(Courbebaisse and Souberbielle, 2011).

Serum $\mathrm{Ca}$ and $\mathrm{P}$ in group 2( with aortic valve calcification ) had a borderline negative correlation which is well known, $\mathrm{P}$ and CaxP product had a highly significant positive correlation $(\mathrm{p}=$ 0.0001) again supporting the opinion of their incrimination in vavlular calcification process.

In group 3( aortic and mitral valve calcification ), parathyroid hormone had a negative correlation with age $(\mathrm{p}=05)$, which supports that it doesn't play much a role in valvular calcification in elderly.

We also found highly significant negative correlation of serum $\mathrm{Ca}$ and $\mathrm{P}$, which is well known. Against what was expected, we found a positive borderline correlation between serum calcium and CaxP product. Also against what was expected a non significant positive correlation was found between serum $\mathrm{P}$ and CaxP product. Does serum $\mathrm{Ca}$ predict $\mathrm{CaxP}$ product level whenever valvular calcification is more extensive or prolonged? This does need further research. We found in group 3( aortic and mitral valve calcification), a highly significant correlation between urea and parathyroid hormone. There was a negative highly significant correlation between CaxP product and creatinine. Also an inverse significant correlation between serum calcium and creatinine level which is well known.

In group 2 (aortic calcification), there was a positive significant correlation between serum calcium level and calcium dose which is predicted and an inverse borderline significant correlation between serum $\mathrm{P}$ and calcium dose. We found an inverse correlation between serum calcium level and alfacalcidiol dose which may mean that calcium is consumed in valve and vascular calcification all over the body.

In group 3( aortic and mitral calcification ), there was an indirect significant correlation between serum $\mathrm{P}$ and calcium dose, which could incriminate the calcium 
as a drug encouraging increases in CaxP product and tissue calcification in ESRD on HD with extensive valve calcification. In our study, patients having aortic calcification were 39 , patients having mitral calcification were 13,5 patienst had aortic regurge while 5 patients had mitral regurge. The rest of our patients had no valvular insufficiency.

In our study, aortic calcification was noted in $65 \%$ dialysis patients. The incidence of valvular insufficiency was $3.9 \%$ in patients with aortic calcification versus $0.63 \%$ in patients without calcification.

Valvular calcification may be associated with aortic regurgitation (Voklov et al., 2009). Ribeiro et al. (1998), found that aortic calcification was noted in $52 \%$ of dialysis patients as compared to $4 \%$ of controls. The prevalence of valvular insufficiency was $22 \%$ in patients with aortic calcifications versus $6 \%$ in patients without calcification.

In our study none of the patients had stenosis of mitral nor aortic valves. Valvular calcification may be associated with stenosis of mitral and aortic valves (Volkov et al., 2009).

Myocardial dysfunction is common just prior to the onset of dialysis (Zoccali et al., 2006 and McCullough et al., 2004).

Ejection fraction was significantly higher in patients with no calcification compared to patients with aortic valve calcification $(\mathrm{p}=0.02)$ and this is expected.

ESD was significantly higher in group 3( aortic and mitral valve calcification ), compared to group 1( no calcification) which means some degree of enlargement of ventricle in group $3(\mathrm{p}=0.02)$. Also in group 3 , ejection fraction and shortening fraction are significantly lower than group $1(\mathrm{p}=0.03$ and 0.005$)$ and this shows the relation between calcification and cardiac dysfunction.

There was no much difference in echocardiographic parameters between group 2( aortic calcification) and group 3( aortic and mitral calcification) and this means that once calcification happends, changes of echo parameters starts.

Cardiovascular calcification is associated with cardiovascular disease and mortality among patients receiving dialysis, and the disorder progresses rapidly once established (Raggi et al., 2011).

In our study, $3 \%$ of patients had left ventricular hypertrophy. Turkmen et al. (2008), studied a total of 82 consecutive patients (52 male, mean age $48 \pm 12$ years) undergoing chronic HD treatment for $>1$ year subjected to echocardiography, left ventricular hypertrophy was detected in 59 (72\%) the study patients, Strozecki et al. (2005) found that valvualr calcification coexisted with left ventricular hypertrophy. Left ventricular hypertrophy (LVM) is the consequence of combined effects of chronic hemodynamic overload and non hemodynamic biochemical and neurohumoral factors characteristic of uremia (Nasri et al., 2004). 
Left ventricular hypertrophy is the most frequent cardiac alteration in ESRD (London et al., 2008).

$\mathrm{LVH}$ has been found in as many as 30 to $45 \%$ of patients with CKD. ( Mizobuchi et al., 2007 ).

In our present study, left atrial enlargement was found in $(7.8 \%)$ the patients.

Kocinaj et al. (2009), analyzed data from 123 patients who were on regular HD, by means of traditional transthoracic echocardiographic examination. The most presented age group was 60 to 90 years old, with predominance of females $(56.1 \%)$. He found dilated left atrium in $26.02 \%$ of study patients. In group 1, we found in our study a direct positive correlation between CaxP and aortic valve dimension $(\mathrm{p}=0.06)$, which was expected.

Group 2( aortic calcification), calcium had an inverse correlation with ejection fraction which was against our expectations $(\mathrm{p}=$ 0.06) also phosphorus had a positive correlation with shortening fraction, $(\mathrm{p}=$ 0.03 ) which was against our expectations.

In group 3, with mitral and aortic calcification, CaxP product was inversely correlated to interventricular septal dimension $(\mathrm{p}=0.03)$. Increase in CaxP product can be associated with decrease in

\section{REFERENCE}

Adragao T (2009). Non invasive evaluation of vascular calcification in CKD patients. KDIGO CKD-MBD work group. Kidney Int; 76 (suppl 113): S1-S130.

Adragao T, Pires A, Lucas C, et al. (2004). A simple vascular calcification score predicts interventricular septal dimension in case of ventricular enlargement. Which may be due to use of high flux dialysers.

Pronounced interstitial fibrosis is a dominant feature of CKD associated structural myocardial remodeling (Ahmed et al., 2007).

$\mathrm{P}$ and CaxP product both had an inverse correlation with posterior wall dimension $(\mathrm{p}$ $=0.08, \mathrm{p}=0.03)$ respectively, this needs more research by cardiologists as both $\mathrm{P}$ and CaxP are incriminated in tissue calcification in ESRD patients on HD.

The extensive intramyocardial fibrosis in ESRD patients with elevated parathyroid dhormoen could be responsible for attenuation of the hypertrophic response to pressure overload and the development of high stress cardiomyopathy and cardiac failure (London et al., 2002).

\section{Conclusion:}

We had a very high incidence of valvular calcification (65\%) as compared to other studies we have to revise our policy towards HD treatment using calcium supplement and $\alpha$-cholecalciferol treatment. Also, we have to revise our policy towards the high flux dialysers now. Phosphorus and CaxP product should be controlled as much as possible.

cardiovascular risk in haemodialysis patients. Nephrol Dial Transplant; 19(6):1480-1488.

Ahmed A, Rich MW, Sanders PW, et al. (2007). Chronic kidney disease associated mortality in diastolic versus systolic heart 
failure: a propensity matched study. Am J Cardiol; 99(3):393-398.

Barasch E, Gottdiener JS, Marino Larsen EK, Chaves PH, Newman AB (2006). Cardiovascular morbidity and mortality in community-dwelling elderly individuals with calcification of the fibrous skeleton of the base of the heart and aortosclerosis (The Cardiovascular Health Study). Am J Cardiol; 97(9):1281-1286.

Bethesda (2004). US renal data system. USRDS 2004 Annual Data report. National Institutes of Health, National Institute of Diabetes and Digestive and Kidney Diseases.

Braun J, Oldendorf M, Moshage W, et al. (1996). Electron beam computed tomography in the evaluation of cardiac calcification in chronic dialysis patients. Am J Kidney Dis; 27(3):394-401.

Courbebaisse M, Souberbielle JC (2011). Phosphocalcic metabolism: regulation and explorations]. Nephrol Ther; 7(2):118-138.

Cozzolino M, Ciceri P, Volpi EM, Olivi L, Messa PG (2009). Pathophysiology of calcium and phosphate metabolism impairment in chronic kidney disease. Blood Purif; 27(4):338-344.

Doumas B, Watson W and Biggs $M$ (1971). Albumin standards and the measurement of serum albumin with bromocresyl green. Clin Chim Acta; 31:87-96.

Endras DB, Villanueva R, Sharp CF Jr, Singer FR (1989). Measurement of parathyroid hormone. Endocrina Metab Clin North Am 18(3): 611-29.

Ernesto U, Ahmed W, Alpert MA (2003). Valvular and perivalvular abnormalities in end-stage renal disease. Am J Med Sci; 325(4):237-242.
Farrel EC (1987). Phosphorus. In: Pesce AJ, Kaplan LA, eds. Methods in clinical chemistry, St Louis: CV Mosby: 1038-1042.

Forman MB, Virmani R, Robertson RM, Stone WJ (1984). Mitral anular calcification in chronic renal failure. Chest; 85(3):367-371

Fox CS, Larson MG, Vasan RS, et al. (2006). Cross-sectional association of kidney function with valvular and annular calcification: the Framingham heart study. J Am Soc Nephrol; 17(2):521-527

Fox E, Harkins D, Taylor H, et al. (2004). Epidemiology of mitral annular calcification and its predictive value for coronary events in African Americans: the Jackson Cohort of the Atherosclerotic Risk in Communities Study. Am Heart J; 148(6):979-984

Huting J (1994). Mitral valve calcification as an index of left ventricular dysfunction in patients with end-stage renal disease on peritoneal dialysis. Chest; 105(2):383-388.

Ikee R, Honda K, Ishioka K, et al. (2010). Differences in associated factors between aortic and mitral valve calcification in hemodialysis. Hypertens Res; 33(6):622-626.

Isidro $B$ and William $G$ (2002). Cardiovascular clacification in endstage Renal disease.Nephrol. Dial Transplant; 17(2): 336339.

Ix JH, Shlipak MG, Katz R, et al. (2007). Kidney function and aortic valve and mitral annular calcification in the Multi-Ethnic Study of Atherosclerosis (MESA). Am J Kidney Dis; 50(3):412-420.

Kahnooj M, Masoomi M, Naderinasab A, et al. (2010). Relationship between calciumphosphorus product and severity of valvular heart insufficiency in patients undergoing chronic hemodialysis. The Journal of Tehran University Heart Center; 5(2): 78-82. 
Katrin U, Jeffrey S, Bryan K, et al. (2010). KDOQI US commentary on the 2009 KDIGO clinical practice Guideline for the diagnosis, Evaluation, and Treatment of CKD-mineral and bone disorder (CKD-MBD). American Journal of Kidney Diseases; 55: 5.

Kocinaj D, Gashi M, et al. (2009). Echocardiographic assessment of left atrial size in patients with end stage renal disease. Cardiovasc J Afr 20(3): 183-6.

London GM (2002). Left ventricular alterations and end-stage renal disease. Nephrol Dial Transplant; 17 Suppl 1:29-36.

\section{London GM, Marchais SJ, Guerin AP, et al.}

(2008). Association of bone activity, calcium load, aortic stiffness, and calcifications in ESRD. J Am Soc Nephrol; 19(9):1827-1835.

Mazzaferro S, Coen G, Bandini S, et al. (1993). Role of ageing, chronic renal failure and dialysis in the calcification of mitral annulus. Nephrol Dial Transplant; 8(4):335340.

McCullough PA, Sandberg KR, Dumler F, Yanez JE (2004). Determinants of coronary vascular calcification in patients with chronic kidney disease and end-stage renal disease: a systematic review. J Nephrol; 17(2):205-215

Mizobuchi M, Finch JL, Martin DR, Slatopolsky E (2007). Differential effects of vitamin D receptor activators on vascular calcification in uremic rats. Kidney Int; 72(6):709-715. Moe M, Drueke T, Block A et al. (2009). KDIGO clinical practice guideline for the diagnosis, evaluation, prevention, and treatment of chronic kidney disease- mineral and bone disorder (CKD-MBD). Kidney International; 76(suppl 113): s22-s49.

Razzaque MS (2011). The dualistic role of vitamin $D$ in vascular calcifications. Kidney Int; 79(7):708-714.

Renoe BW, McDonald JM, Ladenson JH (1980). The effects of stasis with and without exercise on free calcium, various cations and
Moran A, Katz R, Jenny NS, et al. (2008). Left ventricular hypertrophy in mild and moderate reduction in kidney function determined using cardiac magnetic resonance imaging and cystatin $\mathrm{C}$ : the multi-ethnic study of atherosclerosis (MESA). Am J Kidney Dis; 52(5):839-848.

Movilli E, Feliciani A, Camerini $\mathrm{C}$ et al. (2005). A high calcium-phosphate product is associated with high $\mathrm{C}$-reactive protein concentrations in hemodialysis patients. Nephron Clin Pract; 10: 161-167.

Nasri H, Baradaran A, Naderi AS (2004). Close association between parathyroid hormone and left ventricular function and structure in end-stage renal failure patients under maintenance hemodialysis. Acta Med Austriaca; 31(3):67-72

O'Neill WC, Lomashvili KA, Malluche HH, Faugere MC, Riser BL (2011). Treatment with pyrophosphate inhibits uremic vascular calcification. Kidney Int; 79(5):512-517.

Peacock M (2010). Calcium metabolism in health and disease. Clin J Am Soc Nephrol; 5 (1):S23-30.

Raggi P, Boulay A, Chasan-Taber S, et al. (2002). Cardiac calcification in adult hemodialysis patients. A link between endstage renal disease and cardiovascular disease? J Am Coll Cardiol; 39(4):695-701

Raggi P, Chertow GM, Torres PU, et al. (2011). The ADVANCE study: a randomized study to evaluate the effects of cinacalcet plus low-dose vitamin D on vascular calcification in patients on hemodialysis. Nephrol Dial Transplant; 26(4):1327-1339.

related parameters. Clin Chim Acta 103: 91100.

Ribeiro S, Ramos A, Brandao A, et al. (1998). Cardiac valve calcification in haemodialysis patients: role of calcium- 
phosphate metabolism. Nephrol Dial Transplant; 13(8):2037-2040.

\section{Rochelle C, Corretti M and Henrich W} (2010). Valvular Heart Disease in patients with End-stage Renal disease. J Am Soc Nephrol; 20: 388-396.

Rufino M, Garcia S, Jimenez A, et al. (2003). Heart valve calcification and calcium $x$ phosphorus product in hemodialysis patients: analysis of optimum values for its prevention. Kidney Int 85:S115-118

Silver J, Levi R (2005). Regulation of PTH synthesis and secretion relevant to the management of secondary hyperparathyroidism in chronic kidney disease. Kidney Int 95:S8-12

\section{Stinebaugh J, Lavie CJ, Milani RV, Cassidy}

MM, Figueroa JE (1995). Doppler echocardiographic assessment of valvular heart disease in patients requiring hemodialysis for end-stage renal disease. South Med J; 88(1):65-71

Straumann E, Meyer B, Misteli M, Blumberg A, Jenzer HR (1992). Aortic and mitral valve disease in patients with end stage renal failure on long-term haemodialysis. $\mathrm{Br}$ Heart J; 67(3):236-239.

Strozecki P, Odrowaz-Sypniewska G, Manitius J (2005). Cardiac valve calcifications and left ventricular hypertrophy in hemodialysis patients. Ren Fail; 27(6):733738.

Tarrass F, Benjelloun M, Zamd M, et al. (2006). Heart valve calcifications in patients with end-stage renal disease: analysis for risk factors. Nephrology (Carlton); 11(6):494-496.

Tobacco A, Meiattini F, Moda E, et al. (1979). Simplified enzymic/colorimetric serum urea nitrogen determination. Clin Chim 25: 336-7.
Torun D, Sezer S, Baltali M, et al. (2005). Association of cardiac valve calcification and inflammation in patients on hemodialysis. Ren Fail; 27(2):221-226.

Turknen F, Emre A, Ozdemir A, Sevinc C, Erisken E, Yesilcimen K (2008): Relationship between aortic valve sclerosis and left ventricular hypertrophy in chronic hemodialysis patients. Int Urol Nephrol 40 (2): 497-502.

Uhlig K, Berns JS, Kestenbaum B, et al. (2010). KDOQI US commentary on the 2009 KDIGO Clinical Practice Guideline for the Diagnosis, Evaluation, and Treatment of CKDMineral and Bone Disorder (CKD-MBD). Am J Kidney Dis; 55(5):773-799.

Volkov MM, Smirnov AV, Dobronravov VA, et al. (2009). Heart valve calcification in patients with chronic kidney disease. Klin Med (Mosk); 87(6):31-35

Yatzidis H (1974). New method for direct determination of true creatinine. Clinical Chemistry; 20(9): 1131-1134. Yoshida M, Takamatsu J, Yoshida S, et al. (1999). Scores of coronary calcification determined by electron beam computed tomography are closely related to the extent of diabetesspecific complications. Horm Metab Res; 31(10):558-563

Zoccali C, Benedetto FA, Tripepi G, et al. (2006). Left ventricular systolic function monitoring in asymptomatic dialysis patients: a prospective cohort study. J Am Soc Nephrol; 17(5):1460-1465. 


$$
\begin{aligned}
& \text { الملخص العربى المئ } \\
& \text { دراسة تكلس صمامات القلب فى مرضى الفثل الكلوى المعاثين على الديال الدموى المرى }
\end{aligned}
$$

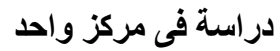

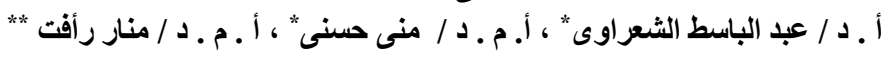

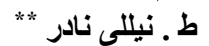

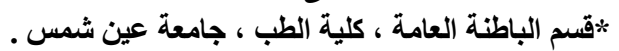

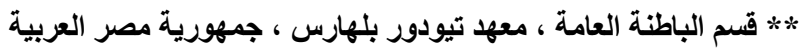

تكلس صمامات القلب أصبح شائعاً بين مرضى الكلى المزمنة ، عوامل الخطورة تتضمن التغيرات فى الكالسيوم و الفسفور ، ارتفاع مستوى ناتج ضرب الكالسيوم والفسفور والارتفاع المستمر فى مستوى هرمون البار اثيرويد فى الام

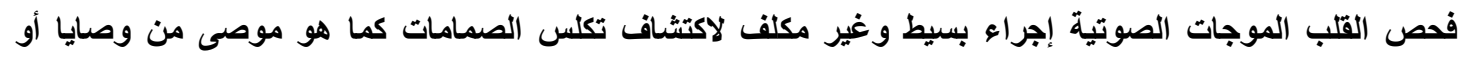
ارشادات KDIGO

المرضى والوسائل 60 مريض معاثين على الديال الدموى كونوا مجموعة ( أ ) ( 36 رجل و24 إمرأة ) و(25 ) من المتطوعين الأصحاء كونوا مجموعة ( ب ) تم تقسيم مجموعة ( أ ) إلى :

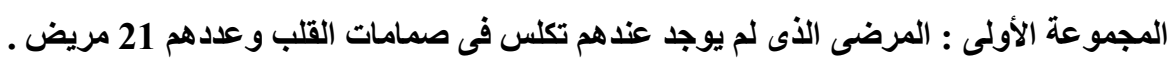
المجموعة الثانية : المرضى الذين يعانون من تكلس فى الصمام الأورطى وعدد هم 26 مريض.

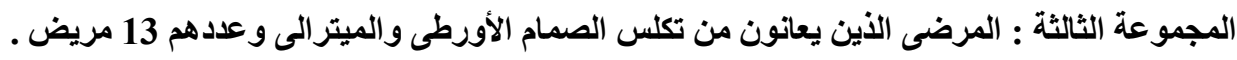

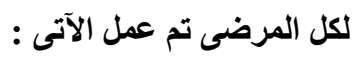

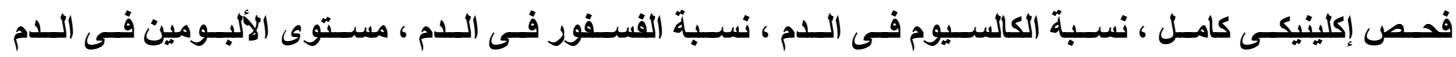
ومستوى هرمون البار اثيرويد فى الام . الموجات الصوتية ثنائية الأبعاد تم عملها لكل المشتركين فى الدراسة وذلك لاكتثاف تكلس صمامات القلب .

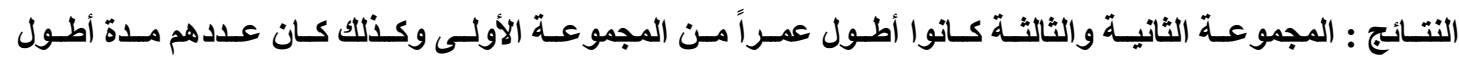

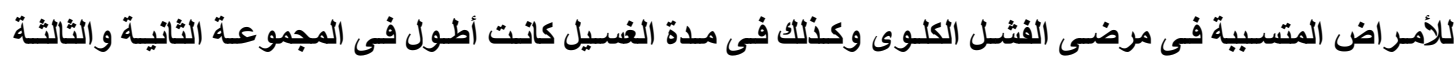

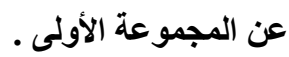
مسـتوى الكالسـيوم كـان أعلـى فـى المجموعـة الثانيـة عـن المجموعـة الأولـى وكــللك فـى المجموعـة الثالثـة عـن

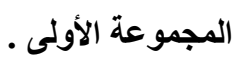
مستوى الفسفوركان أعلى فى المجموعتين الثانية والثالثة عن المجموعة الأولى.

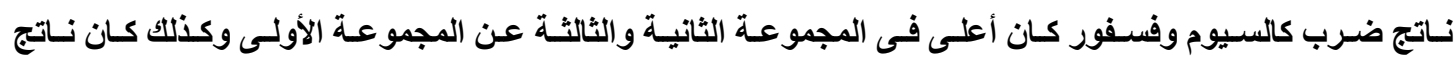
ضرب كالسيوم وفوسفور أعلى من المجموعة الثالثة عن المجموعة الثانية.

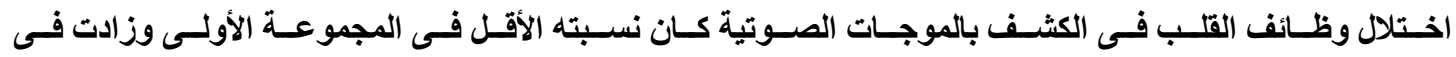
المجموعة الثانية وكاتت الأعلى فى المجموعة الثالثة.

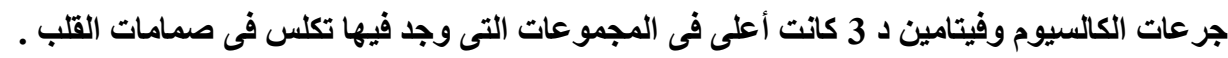

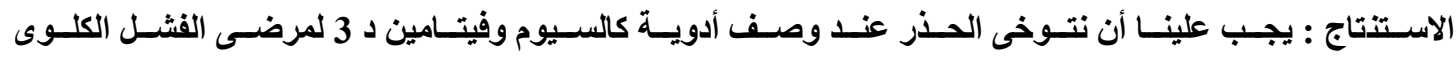
المزمن المعاثين على الديال الاموى. 